\title{
Estado atual das fraturas dos arcos costais
}

\author{
Current state of rib fractures
}

Ricardo Jorge da Silva Pereira', Tatiana Dias de Carvalho², Vitor Engrácia Valenti², Ricardo Araújo³, Sheila Cavalcante Pereira Araújo ${ }^{3}$, Waleska Duarte Melo Albuquerque ${ }^{3}$, Luiz Carlos de Abreu $^{2}$

\section{Resumo}

Os traumas torácicos são uma importante causa de hospitalização, morbidade e mortalidade na população jovem. Em muitas ocasiões, esses traumas torácicos se associam às fraturas dos arcos costais, consideradas benignas na maioria das vezes, mas que podem determinar uma maior gravidade em seus portadores, principalmente idosos. Neste trabalho de revisão, concluiu-se que o diagnóstico correto das fraturas deve ser realizado inclusive com o apoio da tomografia computadorizada, uma vez que a radiologia simples não é suficiente para a precisão diagnóstica. Em relação ao tratamento, muitos autores advogam a terapêutica cirúrgica, objetivando uma recuperação mais rápida dos pacientes internados com fratura de arcos costais.

Palavras-chave: Fraturas ósseas; costelas; fraturas das costelas.

\section{Abstract}

The thoracic trauma is an important cause of hospitalization, morbidity and mortality in young population. In many occasions, this trauma is associated with rib fractures, considered as a benign problem, but they can determine a bigger gravity in its carriers, mainly in elderly. In this review, the authors concluded that the correct diagnosis of the fractures must be carried through with the support of computerized tomography scan, since simple radiology is not enough for the diagnostic precision. In relation to the treatment, many authors defend the surgical approach to obtain a faster recovery of the hospitalized patients with breaking of costal arcs.

Keywords: Fractures, bone; ribs; rib fractures.

Recebido: 16/10/2009

Revisado: $26 / 11 / 2009$

Aprovado: 16/12/2009

\section{Introdução}

Os traumas torácicos são uma importante causa de hospitalização, morbidade e mortalidade na população jovem. Os acidentes de trânsito constituem a principal causa ${ }^{1}$.

Em muitas ocasiões, esses traumas torácicos se associam às fraturas dos arcos costais, consideradas benignas na maioria das vezes. Apesar de promoverem o aparecimento de dor, muitas vezes intensa, consolidam-se bem e raramente promovem complicações, exceto quando decorrentes de traumas de maior energia, nos quais a ocorrência de fraturas de múltiplos arcos costais interfere de forma dramática no quadro respiratório do indivíduo.

A identificação de pacientes com alto risco de morte nos traumas torácicos é fator fundamental nos protocolos de atendimento dos sistemas de trauma. Pacientes com seis ou mais fraturas de costelas são três vezes mais propensos ao óbito dentro das primeiras 24 horas da admissão, quando comparados a pacientes com fratura de apenas uma costela ${ }^{2}$.

A idade e o escore de severidade do trauma são fatores de risco para pneumonia e mortalidade em pacientes com fraturas de costelas

\footnotetext{
Trabalho realizado na Faculdade de Medicina da Universidade Estadual de Ciências da Saúde de Alagoas (Uncisal), Maceió (AL), Brasil

Disciplina de Ortopedia e Traumatologia da Faculdade de Medicina da Universidade Estadual de Ciências da Saúde de Alagoas (Uncisal), Maceió (AL), Brasil

2 Faculdade de Medicina do ABC (FMABC), Santo André (SP), Brasil

${ }^{3}$ Centro Clínico de Ortopedia de Maceió, Maceió (AL), Brasil

Endereço para correspondência: Ricardo Jorge da Silva Pereira - Departamento de Clínica Cirúrgica da Faculdade de Medicina da Universidade

Estadual de Ciências da Saúde (Uncisal) - Rua Doutor Jorge de Lima, 113 - Trapiche da Barra - CEP 57010-382 - Maceió (AL), Brasil -

Tel: (82) 3315-6267 - E-mail: ricardojsp@ig.com.br
} 
ou tórax instável ${ }^{3}$. Assim, o objetivo deste trabalho é descrever os procedimentos adotados em casos de fraturas costais.

\section{Método}

Foi realizada revisão nas bases de dados Medline, Scielo e Lilacs, no período de agosto a setembro de 2009. Os artigos duplicados foram excluídos. Os demais foram selecionados a partir de busca das palavraschave: "fractures and rib and rib fractures". Foram 109 artigos selecionados na busca inicial. Após leitura e seleção dos mesmos, utilizaram-se 29 artigos para composição do desfecho. Somente os artigos que versaram sobre o campo da Ortopedia e Traumatologia foram incluídos na revisão final.

\section{Discussão}

Estudos realizados por Kiøs et al. ${ }^{4}$, em um Centro Regional de Trauma na Noruega, mostraram, numa amostra de 436 pacientes com trauma torácico, que as fraturas de costelas contribuíram com $55 \%$ dos casos, sendo a lesão mais frequentemente encontrada, seguida do pneumotórax, que representou $24 \%$ dos casos.

Sharma et al. ${ }^{5}$ realizaram estudo retrospectivo no período de dez anos em um centro de trauma nível 1 e obtiveram dados em relação às fraturas de costelas. Em relação à idade, Campbell e Dingle ${ }^{6}$ destacaram que cuidados mais expressivos devem ser tomados em relação a pacientes idosos, em que uma simples fratura de costela pode produzir efeitos mais dramáticos.

O diagnóstico de fratura de costelas é realizado pela história do trauma, pela clínica de dor no local da lesão e pela inspiração e dificuldade de movimentação. Na maioria dos serviços de pronto atendimento, o diagnóstico complementar é realizado por meio de uma radiografia simples do tórax ou hemitórax comprometido.

De acordo com Bhavnagri et al. ${ }^{7}$, uma confirmação radiográfica de uma fratura de costela é irrelevante em pacientes que não apresentam alterações clínicas sugestivas de complicações ou traumas associados. Por outro lado, ela é muito importante naqueles que se apresentam sintomáticos e cuja história possa fazer suspeitar de lesão de órgão.

Em diversas ocasiões, porém, as radiografias normais do tórax não evidenciam todas as fraturas de arcos costais, o que pode ser fundamentado pelo recente trabalho apresentado por Ishibashi et $a l .{ }^{8}$, que relataram a importância da tomografia computadorizada tridimensional no diagnóstico de fraturas do esterno e arcos costais, definindo estas como causas de pneumotórax tardio, hemotórax e enfisema subcutâneo maciço. Apresentaram dois casos: no primeiro deles, a radiografia simples mostrou fratura do $8^{\circ}$ arco costal, enquanto a tomografia apresentou fraturas do segundo ao nono arcos costais. No segundo caso, as radiografias normais não mostraram fratura de costelas, enquanto a tomografia evidenciou seis fraturas à direita (do primeiro ao sexto arco) e quatro à esquerda (do primeiro ao quarto arco). Pensamento semelhante têm Livingston et al. ${ }^{9}$ os quais destacaram também a importância da tomografia computadorizada, definindo-a como grande responsável pelo diagnóstico de fraturas não observadas na radiologia convencional e orientando para que esses pacientes com múltiplas fraturas sejam observados, pois são candidatos a insuficiência respiratória.

Traub et al. ${ }^{10}$ defendem que a tomografia computadorizada fornece mais informações diagnósticas no gerenciamento dos traumas torácicos, devendo se constituir exame de rotina na avaliação inicial sempre que possível.

Traumas severos do tórax estão associados a uma significante morbidade e mortalidade. Segundo Esme et al. ${ }^{11}$, a contagem elevada de glóbulos brancos no sangue na admissão, graduação elevada na escala de trauma pulmonar, três ou mais fraturas de costelas e associação com traumatismo cranioencefálico (TCE) contribuem para aumentar esses fatores. Freixinet et al. ${ }^{12}$ destacaram que múltiplas fraturas de arcos costais interferem como indicadoras de severidade no trauma torácico. Lotfipour et al. ${ }^{13}$ consideraram que uma ou mais fraturas unilaterais de costela, além de idade avançada, pressão sistólica inicial $<90 \mathrm{mmHg}$, hemotórax, pneumotórax ou contusão pulmonar constituem efeitos adversos nos pacientes com trauma torácico. Kent et al. ${ }^{14}$ asseguram que a presença de fratura de costelas em pacientes politraumatizados aumenta o risco de mortalidade intra-hospitalar.

Outro fator que deve ser bem avaliado é a presença de enfisema subcutâneo, geralmente benigno, mas que pode ser acompanhado de pneumotórax hipertensivo ${ }^{15}$. Outra complicação relatada por Sata et al. ${ }^{16}$ foi laceração da aorta por fratura cominutiva da nona costela.

O tratamento das fraturas dos arcos costais geralmente é feito de forma conservadora, o que não impede, entretanto, que a decisão por procedimentos cirúrgicos possa ser realizada. No tratamento conservador, os analgésicos e bloqueios são os métodos mais utilizados.

Uma opção de tratamento, proposta por Mohta et al. ${ }^{17}$, preconiza a infusão epidural torácica contínua, através de cateter, com anestésico (bupivacaína). Em trabalho experimental, observaram melhora acentuada da sintomatologia álgica e da função pulmonar. Essa opção foi utilizada durante a permanência do paciente em unidade de terapia intensiva e no ambiente hospitalar. Topçu et al. ${ }^{18}$ também preconizam essa medida terapêutica.

O tratamento cirúrgico, apesar de não ser comum em nosso meio, pode ser indicado em diversas ocasiões e tem como objetivo melhorar a sobrevida do indivíduo traumatizado ${ }^{19}$. Diversos autores propõem, nas situações de múltiplas fraturas de costelas, o tratamento cirúrgico, objetivando a obtenção da estabilidade e melhora do quadro respiratório. Campbell et al. ${ }^{20}$, Konstantinov et al. ${ }^{21}$, Vodicka et al..$^{22}$, Iwasaki et al..$^{23}$, Kakegawa et al..$^{24}$, Richardson et al..$^{25}$, Quell e 
Vécsei ${ }^{26}$ e Meier e Schüpbach ${ }^{27}$ são partidários da fixação das fraturas. Não definiram um tipo específico de fixação, que fica a critério da experiência do profissional.

Nirula et al..$^{28}$ descreveram que os pacientes com traumas torácicos graves, submetidos à intervenção cirúrgica, necessitaram de menos cuidados com relação à ventilação.

As fraturas do esterno são mais raras; porém, $49,6 \%$ dos seus portadores apresentam fratura de costelas, $8 \%$ contusão cardíaca, $4 \%$ trauma da aorta torácica e $2,4 \%$ laceração cardíaca ${ }^{29}$. Muitas vezes vêm acompanhadas de fratura de vértebra torácica, de forma que, na suspeita desta, a coluna vertebral deve ser bem avaliada.

Levando-se em conta que os pacientes portadores de fraturas de costelas ensejam cuidados especiais, pela possibilidade de complicações, e que, na maioria das vezes, são politraumatizados, a fratura de costela está relacionada a um pior prognóstico clínico. A tomografia computadorizada é um exame que sempre deve ser lembrado na busca de fraturas de arcos costais, principalmente porque as radiografias simples não as mostram de forma efetiva.

\section{Referências}

1. Saaiq M, Shah SA. Thoracic trauma: presentation and management outcome. J Coll Physicians Surg Pak. 2008;18(4):230-3.

2. Lien $\mathrm{YC}$, Chen $\mathrm{CH}$, Lin $\mathrm{HC}$. Risk factors for 24-hour mortality after traumatic rib fractures owing to motor vehicle accidents: a nationwide populationbased study. Ann Thorac Surg. 2009;88(4):1124-30.

3. Keel $M$, Meier $C$. Chest injuries - what is new? Curr Opin Crit Care. 2007;13(6):674-9.

4. Kiøs HO, Lande TM, Eriksson U, Nordhaug D, Karevold A, Haaverstad R. [Thoracic injuries at a regional trauma centre]. Tidsskr Nor Laegeforen 2007;127(11):1496-9.

5. Sharma OP, Oswanski MF, Jolly S, Lauer SK, Dressel R, Stombaugh HA. Perils of rib fractures. Am Surg. 2008; 4(4):310-4.

6. Campbell SG, Dingle MA. Rib fractures following minor trauma in older patients: a not-so-benign injury. CJEM. 2000;2(1):32-4.

7. Bhavnagri SJ, Mohammed TL. When and how to image a suspected broken rib. Cleve Clin J Med. 2009;76(5):309-14.

8. Ishibashi H, Ohta SI, Hirose M, Nakajima N. Benefit of three-dimensional computed tomography for traumatic rib fracture: easy to detect and explain rib fractures. Eur J Cardiothorac Surg. 2008;34(2):449.

9. Livingston DH, Shogan B, John P, Lavery RF. CT diagnosis of Rib fractures and the prediction of acute respiratory failure. J Trauma. 2008;64(4):905-11.

10. Traub M, Stevenson M, McEvoy S, Briggs G, Lo SK, Leibman S et al. The use of chest computed tomography versus chest $\mathrm{X}$-ray in patients with major blunt trauma. Injury. 2007;38(1):43-7.

11. Esme H, Solak 0 , Yürümez $Y$, Yavuz $Y$. TThe factors affecting the morbidity and mortality in chest trauma]. Ulus Travma Acil Cerrahi Derg. 2006;12(4):305-10.

12. Freixinet $J$, Beltrán J, Rodriguez $P M$, Juliá G, Hussein $M$, Gil R et al. [Indicators of severity in chest trauma]. Arch Bronconeumol. 2008;44(5):257-62

13. Lotfipour S, Kaku SK, Vaca FE, Patel C, Anderson CL, Ahmed SS et al. Factors associated with complications in older adults with isolated blunt chest trauma. West J Emerg Med. 2009;10(2):79-84.
14. Kent $R$, Woods W, Bostrom 0 . Fatality risk and the presence of rib fractures. Annu Proc Assoc Adv Automot Med. 2008;52:73-82.

15. Perraut M, Gilday D, Reed G. Traumatic occurrence of chest wall tamponade secondary to subcutaneous emphysema. CJEM. 2008;10(4): 387-91.

16. Sata S, Yoshida J, Nishida T, Ueno Y. Sharp rib fragment threatening to lacerate the aorta in a patient with flail chest. Gen Thorac Cardiovasc Surg. 2007:55(6):252-4.

17. Mohta M, Verma P, Saxena AK, Sethi AK, Tyagi A, Girotra G. Prospective, randomized comparison of continuous thoracic epidural and thoracic paravertebral infusion in patients with unilateral multiple fractured ribs $-\mathrm{a}$ pilot study. J Trauma. 2009;66(4):1096-101.

18. Topçu I, Ekici Z, Sakarya M. [Comparison of clinical effectiveness of thoracic epidural and intravenous patient-controlled analgesia for the treatment of rib fractures pain in intensive care unit]. Ulus Travma Acil Cerrahi Derg. 2007;13(3):205-10.

19. Liener UC, Sauerland S, Knöferl MW, Bartl C, Riepl C, Kinzl L et al. [Emergency surgery for chest injuries in the multiply injured: a systematic review]. Unfallchirurg. 2006;109(6):447-52.

20. Campbell N, Conaglen P, Martin K, Antippa P. Surgical stabilization of rib fractures using Inion OTPS wraps-techniques and quality of life follow-up. $J$ Trauma. 2009;67(3):596-601

21. Konstantinov IE, Saxena P, Wood DJ. Stabilisation of chronic flail chest: a novel approach of surgical fixation and osteogenesis. Thorax. 2009;64(3):265-6.

22. Vodicka J, Spidlen V, Safránek J, Simánek V, Altmann P.. [Severe injury to the chest wall - experience with surgical treatment]. Zentralbl Chir. 2007;132(6):542-6.

23. Iwasaki Y, Kawasaki S, Shinozaki M, Yoshimasu T, Okamura Y. [Surgical stabilization of multiple rib fracture and flail chest]. Kyobu Geka. 2006;59(11):980-4.

24. Kakegawa S, Kamiyoshihara M, Ohtaki A, Ohki S, Morishita Y. [Surgical fixation of the ribs for flail chest injuries]. Kiobu Geka. 2006;59(11): 974-9. 
25. Richardson JD, Franklin GA, Heffley S, Seligson D. Operative fixation of chest wall fractures: an underused procedure? Am Surg. 2007; 73(6):591-6.

26. Quell M, Vécsei V. [Surgical stabilization of thoracic wall fractures]. Unfallchirurg. 1991;94(3):129-33.

27. Meier P, Schüpbach P. [Therapy of the unstable thorax in serial fractures of the ribs]. Schweiz Med Wochenschr. 1978;108(16):608-13.

28. Nirula R, Allen B, Layman R, Falimirski ME, Somberg LB. Rib fracture stabilization in patients sustaining blunt chest injury. Am Sur. 2006; 72(4):307-9.

29. Recinos G, Inaba K, Dubose J, Barmparas G, Teixeira PG, Talving P et al. Epidemiology of sternal fractures. Am Surg. 2009;75(5):401-4. 